\title{
Application of Positron Annihilation Spectroscopy Techniques for the Long Range Effect Detection
}

\author{
P. HORODEK ${ }^{a, b}$ \\ ${ }^{a}$ Institute of Nuclear Physics Polish Academy of Sciences, PL-31342 Krakow, Poland \\ ${ }^{b}$ Joint Institute for Nuclear Research, 6 Joliot Curie Str., 141980 Dubna, Russia
}

\begin{abstract}
This paper shows results of conventional positron lifetime and slow positron beam studies of the long range effect. These techniques are not commonly used for this topic. The experimental investigations of pure metals exposed to ion irradiation are reported and discussed on the basis of the literature data. The positron lifetimes measured in the etching experiment give information about kind of defects, their distribution, and existence of the long range effect. The above can be achieved using the positron beam for the case of low energy ion implantation.
\end{abstract}

DOI: 10.12693/APhysPolA.136.361

PACS/topics: $61.80 . \mathrm{Jh}, 61.82 . \mathrm{Bg}, 78.70 . \mathrm{Bj}$

\section{Introduction}

Ion implantation is a commonly known treatment process introducing modification of processed materials. Besides the irradiated surface it largely changes the target structure. This is due to the fact that the implanted ions interact with the material through electron excitations and nuclear collisions, and as a result of losing energy they are retained in the solid. In this way various kinds of lattice defects are produced on ions path. These damages are a subject of various studies $[1,2]$ because of their direct impact on material properties. It was shown that the existence of vacancies can successfully improve absorption rate of hydrogen in palladium being the promising material for hydrogen storages [3]. Moreover, accumulation of structural defects introduced by irradiation in reactors is a main reason for faster wear of materials revealed by e.g. their swelling and enhanced creep [4].

The implanted ions depth distribution depends strictly on the target and ions energy. It is easy to reproduce this distribution by simulations using, e.g. the SRIM/TRIM code [5]. In the simulation defects produced by ions occupy only the implanted area. However, some authors report on existence of so called long range effect (LRE) [6], i.e., damages can be distinguished far behind the implanted depth. For example, Sharkeev et al. [7] found dislocations at the depth of $20 \mu \mathrm{m}$ and $50 \mu \mathrm{m}$ in $\mathrm{Cu}$ implanted with $100 \mathrm{keV} \mathrm{Zr}$ and $\mathrm{Ti}$ ions, respectively. The theoretically predicted ion range was $500 \mathrm{~nm}$. In the stainless steel AISI 316L implanted with $125 \mathrm{keV} \mathrm{N}^{+}$ions Budzynski [8] found decreasing of friction in wear coefficients at the depth up to $1.5 \mu \mathrm{m}$. It was the range 5-fold larger than the implanted area. This observation is explained by the LRE existence caused by movement of not only de-

e-mail: pawel.horodek@ifj.edu.pl fects but also doped atoms. In turn, Lu et al. [9] reported defect clusters distributed to a depth far exceeding the theoretically predicted by SRIM damage range in ionirradiated single crystalline nickel and Ni-based binary. In this case the damage range in nickel was wider than in the alloys.

LRE is an open issue being the subject of studies. So far an unequivocal reason of this effect occurrence has not been given. Sharkeev's investigations $[6,7]$ move forward the idea of generation of local stresses and their extension into the sample. In turn, others seek the reason in diffusion of defects and implanted ions. Additionally, the impact of the kind of material, ion, energy, and fluence on the LRE existence is still unknown.

The convenient method for LRE studies seems to be positron annihilation spectroscopy (PAS). It allows to detect the presence of lattice defects as vacancies and their clusters, dislocations, nanovoids, etc. Depending on the applied PAS technique it is possible to recognize the kind of defects and evaluate their concentration. This method has been successfully used in many fields like e.g. solid state physics or material engineering and applied to various topics and materials [10-12].

In this paper the advantages and limitations of PAS in the study of LRE effect based on two different measurements and data available in literature have been discussed.

\section{Materials and methods}

\subsection{Sample preparation procedure}

Oxygen-free high thermal conductivity (OFHC) copper plates purchased from Nilaco with the dimensions $10 \times 10 \times 1 \mathrm{~mm}^{3}$ have been the objects of studies. They were firstly polished with silicon waterproof abrasive paper and then annealed at $900^{\circ} \mathrm{C}$ for $1 \mathrm{~h}$ in the $\mathrm{N}_{2}$ flow atmosphere. This procedure allowed one to obtain samples containing only residual defects. One sample was saved as a reference. The second specimen was exposed to irradiation at IC 100 cyclotron working 
at the Joint Institute for Nuclear Research (JINR) in Dubna. $\mathrm{Xe}^{26+}$ ions with the energy of about $167 \mathrm{MeV}$ and fluence of $10^{13}$ ions $/ \mathrm{cm}^{2}$ were used. The average $\mathrm{Xe}^{26+}$ flux equalled $5 \times 10^{9} \mathrm{~cm}^{-2} \mathrm{~s}^{-1}$. The temperature during irradiation did not exceed $80^{\circ} \mathrm{C}$.

Similarly, two polycrystalline iron samples of the $99.99 \%$ purity, purchased from Goodfellow, with the dimension of $10 \times 10 \times 1 \mathrm{~mm}^{3}$ were annealed at $1000^{\circ} \mathrm{C}$ for $4 \mathrm{~h}$ in the vacuum conditions of $10^{-5}$ Torr. Then, one plate was saved as the reference, while the second one was exposed to $73 \mathrm{keV} \mathrm{H} \mathrm{H}^{+}$implantation with a fluence of $3 \times 10^{16}$ ions $/ \mathrm{cm}^{2}$ at the UNIMAS implanter [13]. The ion beam current density was not higher than $1 \times 10^{-6} \mathrm{~A} \mathrm{~cm}^{-2}$. Implantation was performed at room temperature with an accuracy of $\pm 3 \mathrm{~K}$. The whole surface of the processed sample was uniformly irradiated.

Ion and vacancy distributions obtained by simulations with SRIM/TRIM code for implanted $\mathrm{Cu}$ and $\mathrm{Fe}$ are shown in Figs. 1a and 2a, respectively.
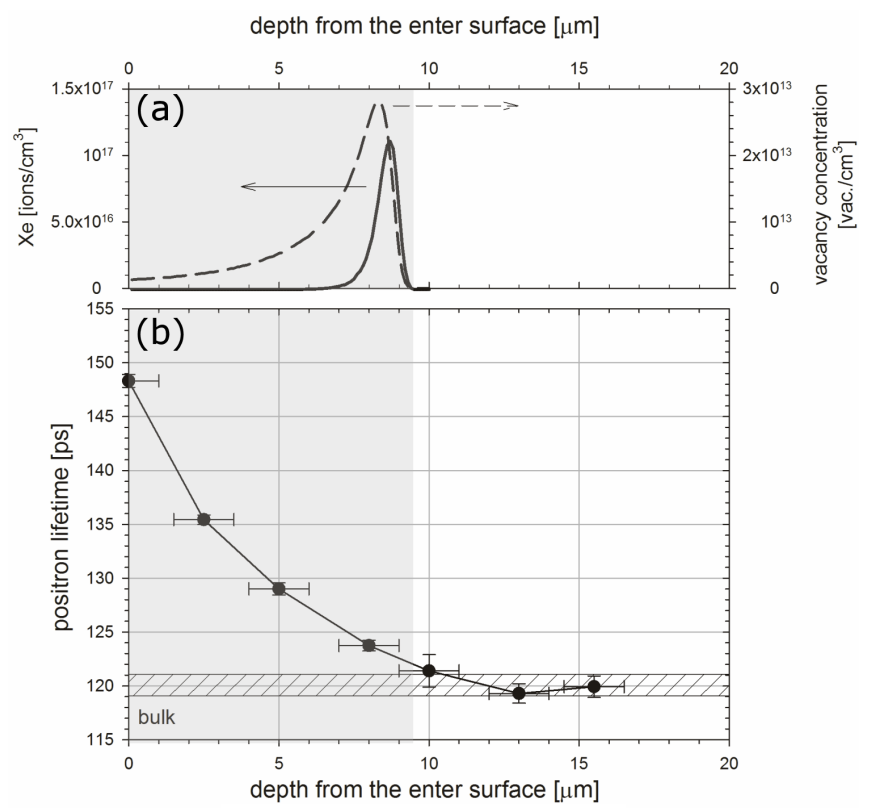

Fig. 1. (a) Results of SRIM calculations of ion (left axis) and vacancy concentration (right axis) as a function of depth after $\mathrm{Xe}^{26+}$ ions implantation with energy of $167 \mathrm{MeV}$ in pure $\mathrm{Cu}$. (b) The depth distribution of positron lifetime component obtained in etching experiment $167 \mathrm{MeV}$. The hatched region represents the value of positron lifetime for well annealed, defect free $\mathrm{Cu}$ sample. Gray region tags the ion implanted layer.

\subsection{Positron measurements}

The PAS experiments can be roughly divided into two groups. The first one represents the measurements performed using positrons implanted into samples after direct emission from the source e.g. Na-22. These positrons are characterized by the continuous energy spectrum and

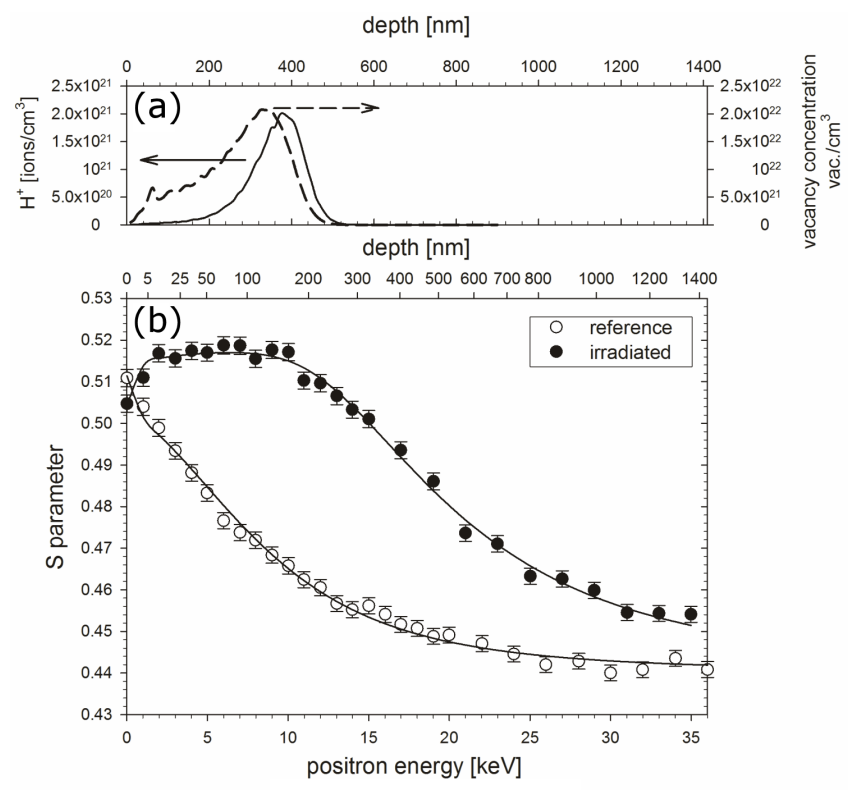

Fig. 2. (a) SRIM calculations of ion (left axis) and vacancy concentration (right axis) as a function of depth after $\mathrm{H}^{+}$implantation with energy of $73 \mathrm{keV}$ in pure Fe. (b) The measured annihilation line shape parameter as a function of the positron incident energy. The top axis means the mean positron implantation depth. The solid lines represent the best fit of diffusion equation to the corresponding experimental points.

they can have energy with different probability from 0 to a maximal energy specific for a given type of isotope. For this reason the mean implantation depth, where about $70-80 \%$ of positrons annihilate, is a few dozen micrometers for $\mathrm{Na}-22$ [14]. In this way mainly materials with defects distributed on the thickness of a few micrometers, like the sample of pure $\mathrm{Cu}$ exposed to $167 \mathrm{MeV}$ $\mathrm{Xe}^{26+}$ irradiation, can be effectively investigated in these experiments.

For investigations of defects distributed on much smaller depths as in the case of a sample of pure Fe implanted with $73 \mathrm{keV} \mathrm{H}^{+}$ions, the second group of PAS experiments is suggested. These are performed using so called variable energy positron beams (VEP). VEP is a flux of monoenergetic positrons implanted with a given energy into a sample. Generally, energy of the beam varies in the range from a few dozen eV up to a few dozen keV. Usually the layers studied with VEP are from hundreds of nanometers up to a few micrometers thick depending on the incident beam energy and kind of target.

Finally, the sample of annealed $\mathrm{Cu}$ and specimen exposed to $167 \mathrm{MeV} \mathrm{Xe} \mathrm{Xe}^{26+}$ irradiation were investigated using the conventional positron lifetime experiment. The results dealing with this experiment presented in the paper were reported in Ref. [15]. They are given here to shed a light how to use PAS for LRE detection. Measurements were performed using the 
fast-fast spectrometer based in $\mathrm{BaF}_{2}$ scintillators with a time resolution of $250 \mathrm{ps}$. The isotope Na-22 with the activity $32 \mu \mathrm{Ci}$ enveloped into a $7 \mu \mathrm{m}$ thick kapton foil was placed between two samples. The LT program [16] was used to analyze the obtained spectra including $10^{6}$ counts.

In turn, unimplanted pure $\mathrm{Fe}$ as well implanted with $73 \mathrm{keV} \mathrm{H}^{+}$ions were examined by Doppler spectroscopy (DB) using VEP at JINR in Dubna. The monoenergetic positron flux with diameter of $3 \mathrm{~mm}$ and intensity $10^{6} \mathrm{e}^{+} / \mathrm{s}$ was used. The maximal energy of implanted positrons was $36 \mathrm{keV}$. The annihilation gamma radiation was registered by a HPGe detector with the energy resolution $1.20 \mathrm{keV}$ interpolated for the energy $511 \mathrm{keV}$. More details about this apparatus are given in Ref. [17]. The obtained DB spectra were analyzed by extraction of $\mathrm{S}$ parameter from the annihilation line. This is defined as a ratio of area under the central part of the $511 \mathrm{keV}$ line to the total area below this peak. This evaluates the participation of positron-electron pairs with a low momentum occurring mostly at open volume defects in the crystalline structure.

\section{Results and discussion}

\subsection{Conventional PAS experiment}

The detection of LRE is strictly related to evaluation of the thickness of damaged layer and its comparison with the theoretically or experimentally estimated ion implanted depth. In the conventional PAS experiments the maximal as well as mean positron implantation ranges are constant and limited. For example, regarding the simulations, $50 \%$ of positrons annihilate at the depth of less than $11.1 \mu \mathrm{m}$ and about $71 \%$ at the depth of about $22.3 \mu \mathrm{m}$ in the case of positrons emitted directly from Na-22 into $\mathrm{Cu}$ [15]. This proves that the range of positron implantation covered the total thickness of ion implanted layer of $\mathrm{Cu}$ sample irradiated with $167 \mathrm{MeV} \mathrm{Xe} \mathrm{Xe}^{26+}$ ions and beyond. It is possible to scan this layer via chemical etching of studied sample. In this way the thin, well defined layer of material is removed and positron lifetimes measured after each etching step. It was shown many times that etching does not introduce additional defects [18, 19].

Given the above irradiated with $167 \mathrm{MeV} \mathrm{Xe} \mathrm{Xe}^{26+}$ ions sample of $\mathrm{Cu}$ was sequentially etched in the 1:3 solution of nitric acid and distilled water. The thickness of about $2-3 \mu \mathrm{m}$ was removed in each step. The accuracy of a digital micrometer screw used in the thickness measurement was equal to $1 \mu \mathrm{m}$.

Positron lifetimes for $\mathrm{Cu}$ samples depending on the depth from the surface are shown in Fig. 1b. The hatched region represents value of positron lifetime of $120 \pm 1$ ps for the non-irradiated, well annealed sample. The single lifetime component obtained for the irradiated specimen reveals existence of only one kind of defects - dislocations. The highest value was registered for the enter surface and it decreased with the increasing depth. At $10 \mu \mathrm{m}$ it reaches the bulk value and marks the end of damaged zone. This corresponds well with the theoretically estimated depth of the implanted layer using the SRIM code and allows to conclude about the lack of LRE [15].

Using the same etching PAS experiment LRE was also invisible in $\mathrm{Ti}$ irradiated with $167 \mathrm{MeV}$ and lower energies $\mathrm{Xe}^{26+}$ ions. Despite the small difference between the theoretically predicted depths and those obtained in measurements there was no reason to conclude about the LRE existence [20]. According to the data reported e.g. by Sharkeev $[6,7]$ the difference should be pronounced. The LRE was successively observed using PAS in $\mathrm{Fe}$ irradiated with the same ions. In this case at the depth of $9 \mu \mathrm{m}$, as pointed by SRIM/TRIM for the implanted layer, huge vacancy clusters were observed. Beyond that, the area occupied by dislocations stretches up to $18 \mu \mathrm{m}$ [21]. In Fe and well annealed $\mathrm{Ag}$ exposed to $\mathrm{Xe}^{26+}$ ions with energies of $18.5,45.5,122.5 \mathrm{MeV}$, PAS measurements allowed one to detect dislocations and clusters of vacancies in $\mathrm{Fe}$, and only dislocations in $\mathrm{Ag}$ in a damaged layer extended beyond the implanted layer. The calculations using a finite element method indicated that the stresses in the implanted layer exceeded the yield strength several times [22].

\subsection{VEP experiment}

The VEP results obtained for reference and implanted with $73 \mathrm{keV} \mathrm{H}^{+}$ions samples of pure Fe are presented in Fig. $2 \mathrm{~b}$ as the $\mathrm{S}$ parameter in the function of positron incident energy. On the top of abscissa the mean positron implantation depth is tagged. It was obtained from the relation

$$
\bar{z}=\frac{A}{\rho} E^{n},
$$

where $\rho=7.86 \mathrm{~g} / \mathrm{cm}^{3}$ is the density of material, $A=2.62 \mu \mathrm{g} \mathrm{cm}^{-2} \mathrm{keV}^{-n}$, and $n=1.692$ as it is for $\mathrm{Fe}[23]$. Therefore the total depth studied by VEP equals approximately $1.4 \mu \mathrm{m}$. The implanted depth according to the SRIM/TRIM calculations is about $500 \mathrm{~nm}$ (Fig. 2a). The white circles in Fig. 2b represent the well annealed, defect-free sample. In this case $\mathrm{S}$ parameter decreases with energy and saturates to the constant value. However, for implanted target (black circles) the $\mathrm{S}$ parameter increases in a short range of energies, then achieves the plateau between 2 and $11 \mathrm{keV}$ and decreases in the direction of $\mathrm{S}$ parameter for the reference sample. The plateau is undoubtedly associated with the presence of implantation defects introduced by $\mathrm{H}^{+}$. To describe the obtained dependencies the VEPFIT code [24] was used. The results of fitting procedure are shown by solid black lines. In the fitting, next to the mentioned $A$ and $n$, Makhov's parameter $m=1.766$ has been used. Positron diffusion length $L_{+}$, obtained for the reference sample, equals $158 \pm 2 \mathrm{~nm}$ and it is in a perfect agreement with the one obtained for the defect-free Fe structure [25]. Shortening of $L_{+}$to only $1 \mathrm{~nm}$ proves the existence of 
open-volume defects with high concentration. The damaged layer thickness estimated on the basis of fitting procedure equalled $361 \pm 14 \mathrm{~nm}$ and it is shorter in comparison to the SRIM/TRIM value of ca. $500 \mathrm{~nm}$. It shows a lack of LRE in this case.

It should be mentioned that the VEP technique was applied for detection of defect range in the implanted samples $[11,12]$. Mazzoldi et al. studied silica glass implanted by $30 \mathrm{keV} \mathrm{Ar}^{+}$[11]. The defect distribution obtained by them was more than twice deeper than the ion range. However, the analysis of positron data in such a case is complicated because the positron diffusion process can mask the defect distribution induced by implantation. Moreover, silicon glass is not a perfect structure itself, as it contains defects and imperfection which can affect the results. Additionally, the mathematical model proposed for analysis of collected data is not perfect. Interpretations are usually provided taking into account the constant values of the Makhov's parameters. However, Ref. [23] shows that they are different for various materials. The commonly used program for fitting $\mathrm{S}(\mathrm{E})$ profiles realizes this procedure only on the basis of the mentioned parameters. Although it allows to use the Makhov's parameters specific for given type of material, it should be stressed that e.g. the Ghosh parameters describe the positron implantation profiles much better, but there is no possibility of using it in any available program [26].

\section{Summary}

The possibility of application of different kinds of PAS experiments for LRE detection has been presented. The conventional one based on the positrons emitted directly from the source can be used for defect profiling of materials exposed to high energy ion irradiation introducing defects on the depths of at least a few micrometers. The sequential etching and measurements depth using micrometric screw makes possible to reproduce the defect profile with the accuracy $\pm 1 \mu \mathrm{m}$.

Experiments based on the VEP tests can be helpful in the case of implantation with relatively small energies introducing defects on the depths of hundreds of nanometers or single micrometers. This method is also convenient for LRE detection, however an inaccurate mathematical model applied for the analysis of the obtained data may cause problems with the precise determination of damaged thickness.

\section{Acknowledgments}

The author expresses his gratitude to J. Dryzek, V.A. Skuratov, and M. Kulik for their help with experiments and ion irradiation.

\section{References}

[1] A. Olejniczak, V.A. Skuratov, Nucl. Inst. Meth. B 326, 33 (2014).

[2] M. Kulik, D. Kołodyńska, A. Bayramov, A. Drozdziel, A. Olejniczak, J. Żuk, Spectrochim. Acta A 198, 222 (2018).

[3] H. Abe, H. Uchida, Y. Azum, A. Uedono, Z.Q. Chen, H. Itoh, Nucl. Inst. Meth. B 206, 224 (2003).

[4] P.T. Heald, M.V. Speight, Acta Metall. 23, 1389 (1975).

[5] J.F. Ziegler, The Stopping and Range of Ions in Solids, Pergamon, New York, 1985.

[6] Y. Sharkeev, E.V. Kozlov, Surf. Coat. Technol. 158, 219 (2002).

[7] Y.P. Sharkeev, N.V. Girsova, A.I. Ryabchikov, E.V. Kozlov, O.B. Perevalova, I.G. Brown, X.Y. Yao, Nucl. Instrum. Meth. B 106, 532 (1995).

[8] P. Budzynski, Nucl. Instrum. Meth. B 342, 1 (2015).

[9] C. Lu, K. Jin, L.K. Béland, F. Zhang, T. Yang, L. Qiao, Y. Zhang, H. Bei, H.M. Christen, R.E. Stoller, L. Wang, Sci. Rep. 6, 19994 (2016).

[10] J. Dryzek, E. Dryzek, B. Cleff, Appl. Surf. Sci. 116, 236 (1997).

[11] E. Dryzek, J. Kuriplach, J. Dryzek, J. Phys. Condens. Matter 10, 6573 (1998).

[12] E. Dryzek, M. Sarnek, K. Siemek, Nukleonika 58, 215 (2013).

[13] M. Turek, S. Prucnal, A. Drozdziel, K. Pyszniak, Nucl. Instrum. Meth. B 269, 700 (2011).

[14] P. Horodek, J. Dryzek, Nukleonika 55, 17 (2010).

[15] P. Horodek, J. Dryzek, V.A. Skuratov, Vacuum 138, 15 (2017).

[16] J. Kansy, Nucl. Instrum. Method. Phys. Res. A 374, 235 (1996).

[17] P. Horodek, A.G. Kobets, I.N. Meshkov, A.A. Sidorin, O.S. Orlov, Nukleonika 60, 725 (2015).

[18] J. Dryzek, E. Dryzek, Tribol. Int. 40, 136 (2007).

[19] J. Dryzek, E. Dryzek, T. Suzuki, R. Yu, Tribol. Lett. 20, 91 (2005).

[20] P. Horodek, J. Dryzek, Surf. Coat. Technol. 355, 247 (2018).

[21] P. Horodek, J. Dryzek, V.A. Skuratov, Rad. Phys. Chem. 122, 60 (2016).

[22] J. Dryzek, P. Horodek, M. Dryzek, Appl. Phys. A 124, 451 (2018).

[23] J. Dryzek, P. Horodek, Nucl. Instrum. Meth. B 266 , 4000 (2008).

[24] A. Van Veen, H. Schut, M. Clement, A. Kruseman, M.R. Ijpma, J.M.M. De Nijs, Appl. Surf. Sci. $\mathbf{8 5}$ 216 (1995).

[25] C.W. He, K. Dawi, C. Platteau, M.F. Barthe, P. Desgardin, S. Akhmadaliev, J. Phys. Conf. Ser. 505, 012018 (2014).

[26] J. Dryzek, P. Horodek, Appl. Phys. A 121, 289 (2015). 